\title{
EFECTO DE UNA FUENTE ALTERNA DE FÓSFORO EN LA PRODUCCIÓN DE CEBOLLA DE BULBO (ALLIUM CEPA L.) BAJO CONDICIONES DE CAMPO
}

\section{EFFECT OF AN ALTERNATE SOURCE OF PHOSPHORUS IN THE YIELD OF ONION (ALLIUM CEPA L.) UNDER FIELD CONDITIONS}

\section{Elberth Hernando Pinzón-Sandoval, 2 Oscar Eduardo Munevar-Garcia, ${ }^{3}$ David Fernando Torres-Hernandez, ${ }^{4}$ Edwin Ferney Cruz-Ruiz}

\footnotetext{
${ }^{1}$ Ingeniero Agrónomo. M.Sc. Fisiología vegetal. Universidad Pedagógica y Tecnológica de Colombia, Grupo GIPSO.

2 Ingeniero Agrónomo, Estudiante de Maestria en Ciencias Agrarias.

${ }^{3}$ Ingeniero Agrónomo, M.Sc. Fisiología Vegetal. Universidad Pedagógica y Tecnologíca de Colombia, Grupo GISSAT.

${ }^{4}$ Ingeniero Agrónomo, Estudiante de Maestria en Fisiología Vegetal. Universidad Pedagógica y Tecnología de Colombia, Grupo Ecofisiología vegetal.
}

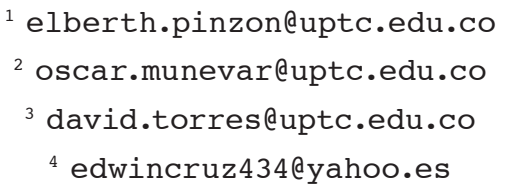

\section{RESUMEN}

La cebolla de bulbo es una de las hortalizas más cultivadas y consumidas a nivel mundial. En Colombia su siembra se concentra en los departamentos de Boyacá y Cundinamarca. Sin embargo, este cultivo en la actualidad presenta problemas de plagas, enfermedades y deficiente nutrición mineral. El buen suministro de macro y micronutrientes es fundamental para que la planta logre desarrollar sus procesos metabólicos adecuadamente. Dentro de los macronutrientes el fosforo $(P)$ tiene un papel relevante ya que este hace parte de la transferencia de energía en los procesos metabólicos y síntesis de las estructuras celulares de las plantas. Por esta razón el objetivo de la investigación fue evaluar una fuente no convencional de fosforo en plantas de cebolla (Allium cepa L.) bajo condiciones de campo. Para esto se empleó un diseño completamente aleatorizado con cuatro tratamientos que consistieron en la aplicación de un fosfato térmico en diferentes porcentajes. Las variables fisiológicas y de crecimiento evaluadas fueron: masa fresca y seca foliar, y de bulbo, área foliar, contenido foliar de fósforo, calcio y magnesio, y rendimiento. Se presentaron diferencias significativas $(P \leq 0,05)$ en las variables masa fresca foliar y de bulbo, masa seca de 
bulbo, área foliar, contenido foliar de magnesio y rendimiento frente a la aplicación de una fuente convencional, esto convierte al fosfato térmico en una alternativa a tener en cuenta dentro de los planes de fertilización de cultivos semestrales dentro de un esquema de nutrición mineral, bajo las condiciones de estudio.

Palabras clave: macroelemento, nutrición mineral, fosfato térmico, hortaliza.

\section{ABSTRACT}

The onion is one of the most cultivated and consumed vegetables worldwide. In Colombia its seed is concentrated in the departments of Boyacá and Cundinamarca. However, this crop currently presents problems of plagues, diseases and low mineral nutrition. The good supply of macro and micronutrients is essential for the plant to develop its metabolic processes properly. Within the macronutrients, phosphorus $(P)$ plays an important role since it is part of the transfer of energy in the metabolic processes and synthesis of the cellular structures of plants. For this reason the aim of the research was to evaluate an unconventional source of phosphorus in onion plants (Allium cepa L.) under field conditions, for which a completely randomized design was used with four treatments that consisted in the application of a thermal phosphate in different percentages The physiological and growth variables evaluated were: fresh and dry foliar, and bulb mass, leaf area, phosphorus, calcium and magnesium foliar content, and yield. There were significant differences $(P \leq 0.05)$ in the variables foliar and bulb fresh mass, bulb dry mass, leaf area, magnesium leaf content and yield versus the application of a conventional source, this converts the thermal phosphate into an alternative to take into account within the fertilization plans of semi-annual crops within a mineral nutrition scheme under the study conditions.

Key words: macroelement, mineral nutrition, thermic phosphate, vegetable.

\section{INTRODUCCIÓN}

En Colombia la cebolla de bulbo (Allium cepa L.) es una de las hortalizas de mayor importancia en los sistemas de producción de clima frio tropical. El departamento de Boyacá cuenta con el distrito de riego del Alto Chicamocha en el que se concentran cerca de 2.500 ha destinadas a la producción de cebolla de bulbo y en el cual se presentan los mejores rendimientos a nivel nacional (Gutiérrez et al., 2013). En el 2017, Boyacá se consolidó como el mayor productor de cebolla de bulbo en Colombia con una participación en el mercado del 56,2\%, un área sembrada de 6.371 ha, una producción de $180.129 \mathrm{t}$, y un rendimiento promedio de 28,1 t. ha-1 (MADR, 2018).
La cebolla de bulbo es una hortaliza muy apetecida por los consumidores debido a sus diferentes formas de presentación en la que se puede encontrar en el mercado que van desde en fresco hasta encurtidos o deshidratada. Lo anterior la posiciona como una de las hortalizas con mayor importancia económica en el mundo (Estrada-Prado et al., 2015). En Colombia, este sistema productivo es un generador de empleo e ingresos especialmente para productores de zonas como el altiplano cundiboyacense en las que se concentra cerca del $68,6 \%$ de la producción nacional (Álvarez-Herrera et al., 2017).

A pesar de ser un cultivo tan importante, presenta bajos rendimientos en comparación con 
otros países de la región (Álvarez-Herrera et al., 2017). Debido a la baja adopción de tecnología en los últimos años y a diferentes problemas dentro de los que se encuentran plagas, enfermedades, uso ineficiente del recurso hídrico y planes inadecuados de nutrición mineral. En Colombia tradicionalmente la nutrición del cultivo se realiza sin un diagnóstico integral previo que conlleva básicamente a la aplicación de fertilizantes convencionales de forma edáfica, sin tener en cuenta los requerimientos del cultivo (Gomez et al., 2010).

El proceso de nutrición mineral de los cultivos juega un papel muy importante ya que, a partir de un adecuado suministro de macro y micronutrientes basado en los requerimientos y su relación con la fenología se podrá aportar de una forma significativa en la expresión del potencial genético de producción de las plantas (Backes et al., 2018). Dentro de los macroelementos, el fosforo $(P)$ tiene un papel relevante ya que hace parte de la transferencia de energía en los procesos metabólicos y la síntesis de las estructuras celulares de las plantas (Marschner, 2012). En consecuencia, la disponibilidad de este elemento tiene un efecto directo sobre el crecimiento y desarrollo de todos los cultivos (Wyngaard et al., 2016). Las fuentes de fósforo empleadas en la agricultura provienen de un recurso de tipo mineral conocido como roca fosfórica el cual es limitado y no renovable (Gilbert, 2009; Zhu et al., 2018).

En la actualidad existe la percepción general de que los fertilizantes convencionales son ineficientes en el aporte de fósforo a los cultivos (Roberts \& Johnston, 2015). Sin embargo, existen fuentes no convencionales como el fosfato térmico con propiedades de mayor disponibilidad debido al proceso de rompimiento térmico de la roca fosfórica, esto genera un material con una solubilidad intermedia que mejora la disponibilidad a corto y largo plazo y ofrece a la planta un mayor porcentaje de fósforo, en forma asimilable durante todo su ciclo fenológico (Fernández \& Noguera, 2003). Dichas propiedades han sido evaluadas en cultivos como Fríjol cv 'ICA Cerinza' (Quintana-Blanco et al., 2017) y Fresa cv 'Albión' (Galindo-López et al., 2018) en los que se observó un mejor desempeño en variables de crecimiento y producción.

Por esto, el objetivo de esta investigación fue evaluar una fuente no convencional de fósforo (fosfato térmico) y su respuesta en variables de crecimiento y producción en el cultivo de cebolla de bulbo (Allium cepa L.) bajo condiciones ambientales y edáficas del municipio de Tibasosa-Boyacá.

\section{MATERIALES Y MÉTODOS}

La investigación se realizó bajo condiciones de campo, en un predio ubicado en el municipio de Tibasosa, Boyacá, con coordenadas 5०44'53"N 72059'56"O y una altura de 2.538 msnm. Las condiciones climáticas durante el desarrollo del experimento fueron: temperatura promedio de $13,9{ }^{\circ} \mathrm{C}$, humedad relativa promedio del $82 \%$ y una precipitación media mensual de $81,7 \mathrm{~mm}$.

Para evaluar el efecto del fosfato térmico se reemplazó parcialmente la fuente de fósforo empleada por el agricultor de forma convencional, para esto se empleó un diseño completamente al azar, con cuatro tratamientos así: T1: $100 \%$ fosfato Diamónico (DAP) (fuente convencional); T2: $80 \%$ DAP, $20 \%$ fosfato térmico (FT); T3: $40 \%$ DAP, $60 \%$ FT y T4: $20 \%$ DAP, 80 \% FT. Los porcentajes se ajustaron al requerimiento del cultivo de acuerdo a lo reportado por Guerrero-Riasco (1995) y las condiciones del suelo determinadas mediante análisis fisicoquímico (Tabla I), se utilizó una fuente comercial de fosfato térmico, con una composición garantizada de $\mathrm{P}_{2} \mathrm{O}_{5} 20$ \%; $\mathrm{CaO} 28$ \%; MgO 8,5 \%; SiO, 15 \%. Cada 
tratamiento se replicó tres veces, para un total de 12 unidades experimentales. Para el establecimiento de las unidades experimentales se tomó una cama de $100 \mathrm{~m}^{2}$, dividida en parcelas de $8 \mathrm{~m}^{2}$, con un total de 225 plantas por parcela. La siembra se realizó por trasplante, se empleó como material vegetal el hibrido Yellow Granex F1.

Para medir las variables se seleccionaron 10 plantas del centro de cada unidad experimental con el fin de evitar el efecto de borde. La aplicación de los tratamientos se hizo al momento de la siembra junto con los demás nutrientes, empleando urea como fuente de nitrógeno, $\mathrm{KCl}$ como fuente de potasio y un producto comercial que garantizara el suministro de elementos menores. Se aplicó riego por aspersión según la necesidad del cultivo, se realizó manejo fitosanitario con base en monitoreo pero respetando las prácticas de manejo del agricultor. La investigación se realizó en el segundo semestre del 2017 con una duración de 120 días a partir del trasplante.

Tabla 1. Análisis físico-químico de suelo, sitio de investigación.

\begin{tabular}{l|c|c} 
Propiedad & Valor & Interpretación \\
\hline $\mathrm{pH}$ & 5,16 & Fuertemente acido \\
\hline $\mathrm{M} . \mathrm{O}(\%)$ & 2,32 & Baja \\
\hline $\mathrm{P}$ Bray II $(\mathrm{ppm})$ & 53,4 & Alto \\
\hline $\mathrm{Ca}\left(\mathrm{cmol} \mathrm{kg} \mathrm{k}^{-1}\right)$ & 7,85 & Alto \\
\hline $\mathrm{Mg}(\mathrm{cmol} \mathrm{kg}$ & Medio \\
\hline $\mathrm{K}(\mathrm{cmol} \mathrm{kg})$ & 1,9 & Alto \\
\hline $\mathrm{Na}(\mathrm{cmol} \mathrm{kg-1})$ & 1,86 & Medio \\
\hline $\left.\mathrm{CE}(\mathrm{dS} \mathrm{m})^{-1}\right)$ & 0,28 & Medio \\
\hline Textura & 0,52 & Franco areno arcilloso
\end{tabular}

Las variables fisiológicas y de crecimiento evaluadas fueron: masa fresca y seca foliar y de bulbo, mediante una balanza electrónica Acculab VIC 612 de 0,01g de precisión y secadas en una estufa Memmert a $70^{\circ} \mathrm{C}$ hasta alcanzar peso constante (Aprox. 72 horas); concentración foliar de fósforo, calcio y magnesio a través del método de calcinación a $600{ }^{\circ} \mathrm{C}$, digestión acida y valoración por espectrofotometría visible llevada a cabo por el laboratorio $A G R I L A B \circledR$. En cuanto al rendimiento, se cuantificó extrapolando los datos de peso fresco de bulbo de la zona muestreada $\left(8 \mathrm{~m}^{2}\right)$ a t.ha-1, se clasificaron los bulbos por su diámetro dentro de los rangos de calidad establecidos por la norma técnica Colombiana NTC 1221 teniendo para la calidad 1
(C1), bulbos de calibre de 71 a $90 \mathrm{~mm}$ y para la calidad 2 (C2), bulbos de calibre de 41 a $70 \mathrm{~mm}$. Las variables de crecimiento y producción fueron cuantificadas a los 120 días después del trasplante.

Los datos obtenidos fueron sometidos a pruebas de normalidad y homogeneidad de varianza mediante las pruebas de Shapiro-Wilk y Levene, respectivamente. Comprobados los supuestos se realizó análisis de varian$z a$, las variables que mostraron diferencias estadísticas fueron sometidas a pruebas de comparación de medias de Tukey ( $P \leq 0,05)$. Los análisis se realizaron con el programa estadístico SAS v.9.2e SAS (Institute Inc., Cary, NC). 
RESULTADOS Y DISCUSIÓN

\section{MASA FRESCA Y SECA FOLIAR Y DE BULBO}

El fosfato térmico empleado generó una repuesta positiva sobre la ganancia de masa fresca en plantas de $A$. cepa con diferencias significativas $(P \leq 0,05)$ entre tratamientos. La aplicación de fosfato térmico en un porcentaje de $80 \%$ en relación al requerimiento del cultivo presentó el mejor resultado de masa fresca foliar y de bulbo con valores de 91,2 $\pm 3,4$ y $155,8 \pm 2,01 \mathrm{~g}$ respectivamente. Por su parte el tratamiento convencional $100 \%$ DAP presentó valores de 39,1 $\pm 6,06$ y 120,4 \pm 5,25 respectivamente (Figura 1 ).

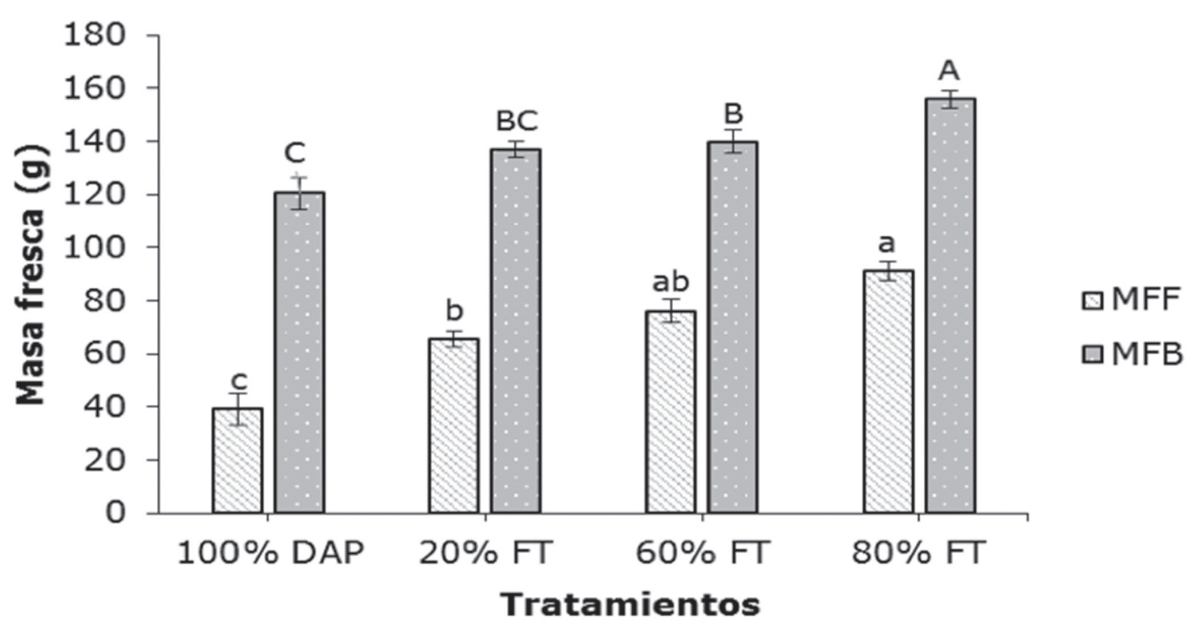

Figura 1. Masa fresca de plantas de cebolla de bulbo (Allium cepa L.) con aplicación de un fosfato térmico en diferentes porcentajes. Letras diferentes en cada serie indican diferencias significativas según la prueba de Tukey $(P \leq 0,05)$. Barras verticales indican error estándar $(n=3)$. MFF: Masa fresca foliar; MFB: Masa fresca de bulbo.

El fósforo es parte de moléculas como el ATP y de la formación de membranas celulares, ya que hace parte de los fosfolípidos (Marschner, 2012), lo cual se verá reflejado, en principio, en la adecuada formación de raíces y por consiguiente mejor toma de agua y nutrientes, esto responde a lo encontrado en el presente experimento en el que la aplicación de un fosfato térmico en un porcentaje del 80 $\%$ generó una mayor masa fresca foliar y de bulbo, posiblemente debido a que este fertilizante al ser citrosoluble e hidrosoluble genera una disponibilidad del elemento de forma más prolongada, ajustándose a los requerimientos de las diferentes etapas fenológicas de la planta (Quintana-Blanco et al., 2017).

La masa seca del bulbo presentó diferencias significativas $(P \leq 0,05)$, mientras que la masa seca foliar no mostró diferencias estadísticas. El tratamiento de 80 \% de fosfato térmico generó un aumento del peso seco del bulbo con un valor de 17,2 $\pm 0,6 \mathrm{~g}$ frente a la aplicación de DAP que presentó un valor de 12,4 $\pm 0,4 \mathrm{~g}$ (Figura 2). 


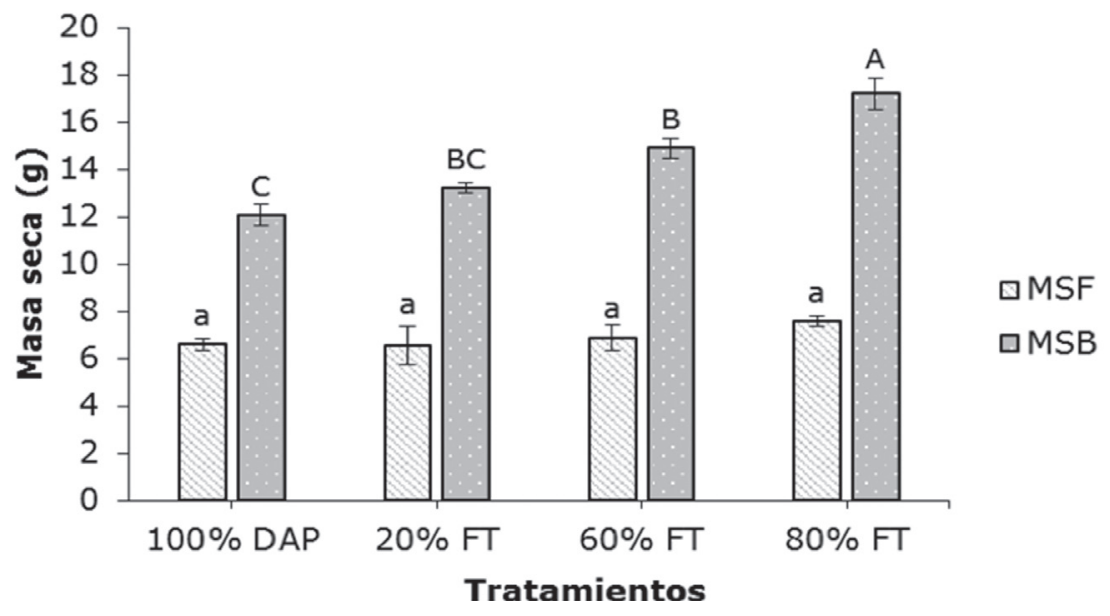

Figura 2. Masa seca en plantas de cebolla de bulbo (Allium cepa L.) con aplicación de un fosfato térmico en diferentes porcentajes. Letras diferentes en cada serie indican diferencias significativas según la prueba de Tukey

$(P \leq 0,05)$. Barras verticales indican error estándar $(n=3)$. MFF: Masa seca foliar; MFB: Masa seca de bulbo.

La masa seca de los bulbos es un factor muy importante para la industria de cebollas deshidratadas, ya que bulbos con poco peso seco son poco apetecidos al mayor gasto de energía para su secado (Bonza-Espinoza et al., 2016). La masa seca de los bulbos determina atributos de calidad como la pungencia y la vida útil en anaquel (Hendriksen \& Hansen, 2001). El fósforo está directamente relacionado con el cotransporte de las triosas fosfatadas del cloroplasto al citosol, donde se sintetizan a sacarosa para su posterior transporte y almacenamiento en los órganos de reserva como almidón, de esta forma se aumenta la masa seca de los órganos vertedero.

\section{ÁREA FOLIAR}

El área foliar presentó diferencias significativas entre tratamientos $(P \leq 0,05)$. La aplicación de un fosfato térmico en diferentes porcentajes generó un aumento del área foliar alcanzando un valor de 458,8 $\pm 8,4 \mathrm{~cm}^{2} /$ planta, en relación con el tratamiento convencional que obtuvo un área foliar de 362,7 $\pm 10,9 \mathrm{~cm}^{2} /$ Planta (Figura 3).

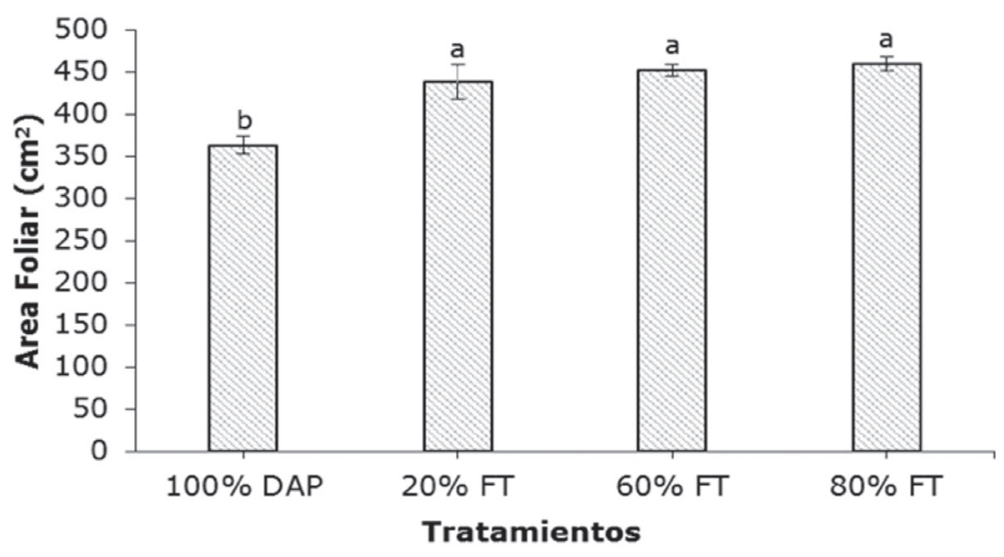

Figura 3. Área foliar de plantas de cebolla de bulbo (Allium cepa L.) con aplicación de un fosfato térmico en diferentes porcentajes. Letras distintas entre tratamientos indican diferencias significativas según la prueba de Tukey $(P \leq 0,05)$. Barras verticales indican error estándar $(n=3)$. 
Cuando el fósforo es limitante o su suministro es deficiente, la planta puede presentar una reducción en la expansión de la hoja y el área foliar, así como también variación en el número de hojas (IPNI, 1999). El área foliar está estrechamente relacionada con la dinámica de la acumulación de materia seca, fósforo y otros nutrientes (Marschner, 2012), siendo la lámina foliar la parte más importante del aparato fotosintético de la planta (Pineda-Mares et al., 2001). Es importante tener en cuenta que el fosfato térmico, dentro de su composición, además de fósforo contiene magnesio y silicio, los cuales son elementos que contribuyen de forma significativa en la síntesis de clorofila, así como en la protección del sistema fotosintético, situación que se verá reflejada en un mayor crecimiento de la planta (Epstein, 2009; Cakmak \& Yazici, 2010).

\section{CONTENIDO DE FÓSFORO, CALCIO Y MAGNESIO EN TEJIDOS}

El contenido de fósforo a nivel de tejido foliar no presentó diferencias significativas $(P \leq 0,05)$, sin embargo los tratamientos con aplicación de fosfato térmico en algún porcentaje se mostraron estadísticamente similares con valores que van desde 0,22 \pm 0,02 hasta 0,26 $\pm 0,005 \%$, en comparación con el tratamiento testigo, el cual presentó un valor de 0,25 $\pm 0,008 \%$ (Figura 4). Lo anterior indica que el fosfato térmico como fuente de fósforo presenta buenas características de solubilidad y disponibilidad para la planta, siendo una excelente alternativa ya que desde el punto de vista económico es una fuente que presenta un valor comercial 2,5 veces menor que la fuente de fósforo empleada convencionalmente.

En la mayoría de plantas la concentración de fósforo que se considera adecuada a nivel de tejido es de 0,2 \% en relación al peso seco (Marschner, 2012), valor que se ajusta a lo observado en la presente investigación. Los valores de fósforo foliar encontrados en los tratamientos de fosfato térmico se pueden asociar al aumento de masa fresca y seca ya que un adecuado nivel de fósforo a nivel foliar tiene un efecto directo en la capacidad fotosintética (Singh et al., 2013), esto genera un correcto crecimiento de las plantas.

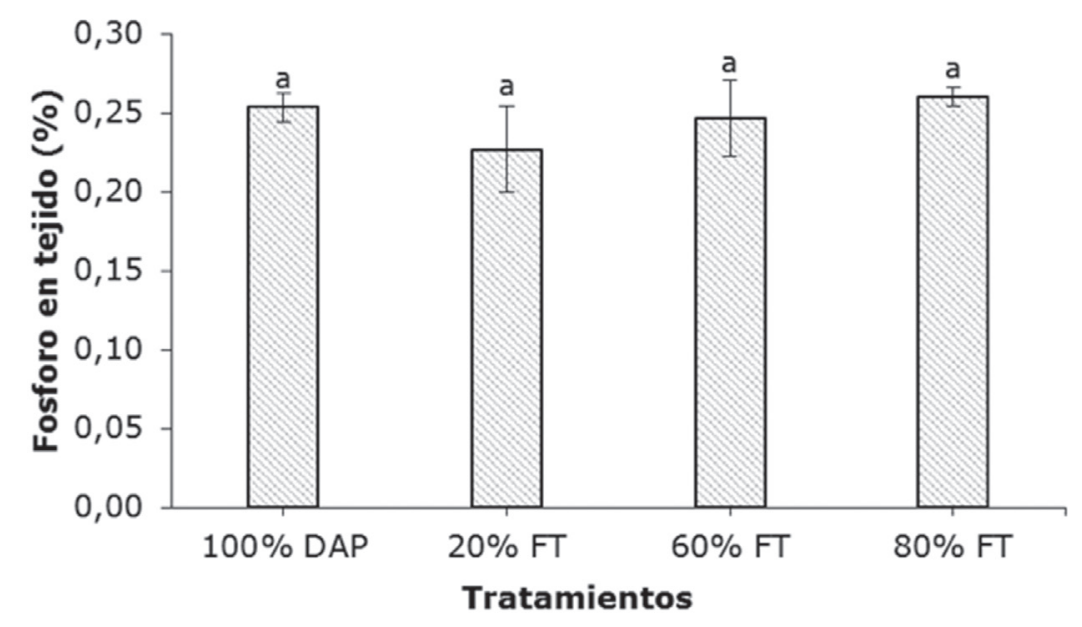

Figura 4. Contenido de fosforo foliar de plantas de cebolla de bulbo (Allium cepa L.) con aplicación de un fosfato térmico en diferentes porcentajes. Letras distintas entre tratamientos indican diferencias significativas según la prueba de Tukey $(P \leq 0,05)$. Barras verticales indican error estándar $(n=3)$. 
El fosfato térmico, al tener silicio en su composición, facilita la disponibilidad de fósforo debido a que los sitios de fijación de este elemento pueden ser ocupados parcialmente por el ácido silícico, quedando el $\mathrm{P}$ en la solución del suelo. Estas reacciones de adsorción-desorción son reversibles y constituyen un mecanismo fundamental para mejorar la disponibilidad de $\mathrm{P}$ en los suelos, generando una mejor dinámica nutricional (Datnoff, 2005).

El contenido de calcio no presentó diferencias estadísticas ( $P \leq 0,05)$; sin embargo, al igual que el fosforo, mostró valores similares al tratamiento testigo (Figura 5A). Los valores observados se ajustan a lo reportado por Larcher (2003) quien indica que el calcio, a nivel foliar, puede oscilar entre 0,04 a 1,3\% en relación al peso seco. Lo anterior indica que el calcio aportado por el fosfato térmico genera un aporte efectivo para la planta, el cual cumplirá funciones de tipo estructural, pues hace parte de las pectinas a nivel de la pared celular, siendo indispensable para la formación de las nuevas células (Marschner, 2012).

Por su parte el contenido de magnesio foliar mostró diferencias significativas $(P \leq 0,05)$. El tratamiento en el que se empleó un 80 $\%$ de fosfato térmico presentó un valor de $0,18 \pm 0,005 \%$, por su parte el tratamiento testigo generó un valor de 0,14 \pm 0,003 \% (Figura 5B). El producto empleado mostró un aporte importante de magnesio, siendo fundamental para el buen funcionamiento del proceso fotosintético de las plantas, debido a que un $75 \%$ del magnesio foliar está involucrado en la síntesis de proteínas y entre el 15 y $20 \%$, asociado con pigmentos de clorofila (White, 2009). Siendo también cofactor de una serie de enzimas involucradas en la fijación fotosintética de carbono (Maathuis, 2009). La respuesta encontrada en variables como área foliar y masa fresca y seca se asocian de una forma clara al aporte de fósforo, calcio y magnesio realizado por el fosfato térmico empleado en el presente estudio.
A

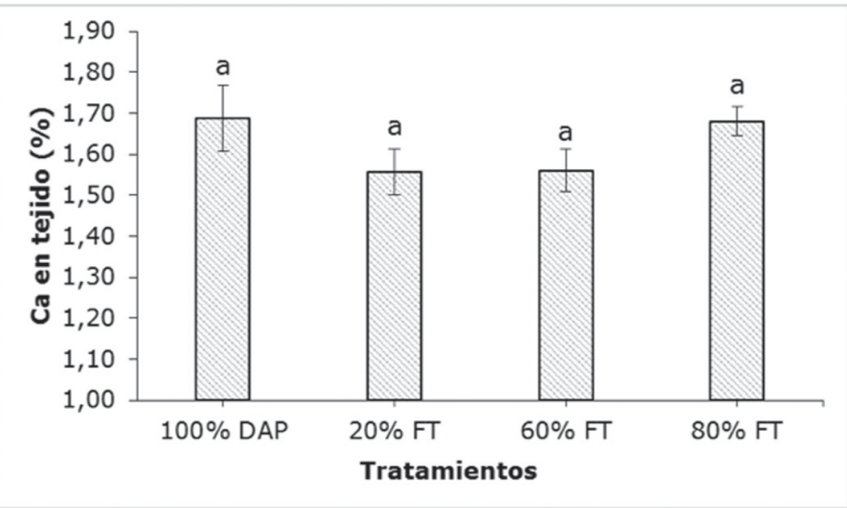

B

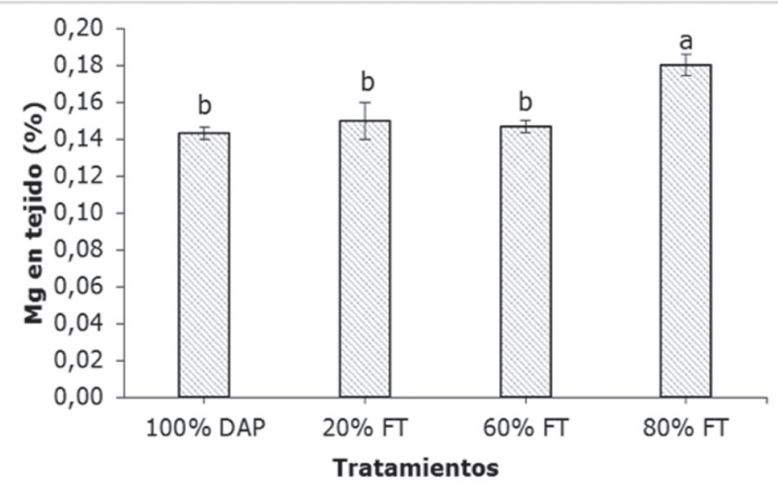

Figura 5. Concentración foliar de A: Calcio; B: Magnesio en plantas de cebolla de bulbo (Allium cepa L.) con aplicación de un fosfato térmico en diferentes porcentajes. Letras distintas entre tratamientos indican diferencias significativas según la prueba de Tukey $(P \leq 0,05)$. Barras verticales indican error estándar $(n=3)$. 


\section{RENDIMIENTO}

La variable rendimiento presentó diferencias significativas $(P \leq 0,05)$ para la clasificación C1 que corresponde a bulbos con un diámetro ecuatorial entre 71 y 90 mm (ICONTEC, 1994). La aplicación del fosfato térmico en diferente porcentaje generó valores entre $57,5 \pm 0,2$ y
$62,2 \pm 0,08 \mathrm{t} \mathrm{ha}^{-1}$, mientras que el tratamiento testigo el cual tuvo como fuente de fosforo DAP mostró un valor de 53,6 \pm 0,5 t ha-1. La clasificación C2 que correspondió a bulbos con diámetro ecuatorial de 41 a $70 \mathrm{~mm}$ no mostró diferencias entre tratamientos (Figura 6).

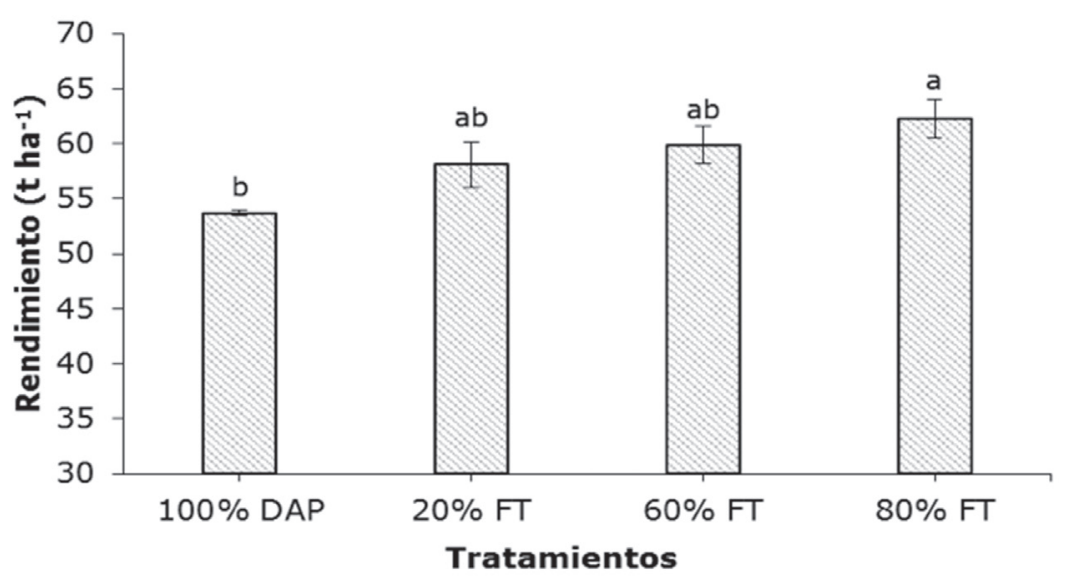

Figura 6. Rendimiento por calidades de plantas de cebolla de bulbo (Allium cepa L.) con aplicación de un fosfato térmico en diferentes porcentajes. Letras distintas entre tratamientos indican diferencias significativas según la prueba de Tukey $(P \leq 0,05)$. Barras verticales indican error estándar $(n=3)$. C1: Bulbos con diámetro de 71 a 90 mm; C2: Bulbos con diámetro de 41 a $70 \mathrm{~mm}$.

El papel del fósforo es esencial en muchos aspectos del metabolismo celular, que van desde el desarrollo embrionario, la germinación, el crecimiento de las plántulas, y la formación del sistema radical, hasta la floración (Marschner, 2012). La aplicación de una fuente alterna de fósforo en las concentraciones usadas en esta investigación resultó en un aumento del rendimiento con respecto a la aplicación de una fuente convencional. Estos resultados coinciden con lo reportado por Quintana et al., (2017) quienes reportan un aumento en el rendimiento de frijol cv 'ICA Cerinza' con la aplicación de una dosis de $600 \mathrm{~kg} \mathrm{ha}^{-1}$ de termofosfato. Por su parte, Fernadez \& Meza (2004) indican que la aplicación de roca fosfórica calcinada como es el caso del fosfato térmico empleado, generó una mejor disponibilidad de fósforo en el cultivo de maíz, representado en una mayor producción de biomasa en un suelo tropical ácido, esto concuerda con los resultados observados en el presente estudio.

\section{CONCLUSIONES}

La aplicación de un fosfato térmico comercial generó una respuesta positiva en variables como masa fresca foliar y de bulbo, masa seca de bulbo, área foliar, además de un resultado similar en la concentración foliar de fósforo, calcio y magnesio y rendimiento t.ha-1 en plantas de cebolla "Allium cepa L." bajo condiciones de campo, frente a la aplicación de una fuente convencional. Esto convierte a esta fuente no convencional de fósforo en una alternativa que 
se puede tener en cuenta dentro de los planes de fertilización de cultivos semestrales dentro de un esquema de nutrición mineral del cultivo de cebolla bajo las condiciones de estudio.

\section{LITERATURA CITADA}

Álvarez-Herrera, J. G., Alvarado-Sanabria, O. H., \& Suesca-Ochoa, F. A. (2017). Efecto de diferentes láminas de riego en el crecimiento y desarrollo de cebolla de bulbo (Allium cepa L.). Revista Colombiana de Ciencias Hortícolas, 11(2), 359367. https://doi.org/http://dx.doi.org/10.17584/ rcch.2017v11i2.7345

Backes, C., Villas, R., Grava, L., Forlan, P., \& Marques, A. (2018). Determination of growth and nutrient accumulation in bella vista onion. Caatinga, 31(1), 246-254.

Bonza-Espinoza, M., Pinzón-Sandoval, E. H., \& Álvarez-Herrera, J. G. (2016). Efecto del nitrato de potasio y la sacarosa sobre el rendimiento de plantas de cebolla (Allium cepa L.). Temas Agrarios, 21(2), 40-50.

Cakmak, I., \& Yazici, A. (2010). Magnesio: El elemento olvidado en la producción de cultivos. Informaciones Agronómicas-IPNI, 94(2), 23-25.

Datnoff, L. E. (2005). Silicon in the Life and Performance of Turfgrass. Applied Turfgrass Science, (August), 12-16.

Epstein, E. (2009). Silicon: Its manifold roles in plants. Annals of Applied Biology, 155(2), 155-160.

Estrada-Prado, Wilfredo, Lescay-Batista, AlvarezFonseca, Alexander, Maceo-Ramos, Y. C. (2015). Niveles de humedad en el suelo en la producción de bulbos de cebolla. Agron. Mesoam., 26(1), 111-117.

Fernadez, S. M., \& Meza, C. A. (2004). Efecto residual de la roca fosfórica de riecito modificada por calcinación o acidulación sobre plantas de maíz en suelos con nivel variable de calcio. Bioagro, 16(2), 93-98.

Fernández, S., \& Noguera, R. (2003). Producción de fosfatos térmicos a partir de rocas fosfóricas nacionales. Agricultura Tropical, 53(1), 49-58.

Galindo-lópez, F., Pinzón-sandoval, E. H., Quintanablanco, W. A., Serrano, P. A., \& Galán, M. (2018). Evaluación de un termofosfato en el cerecimento $\mathrm{y}$ producción de fresa ( Fragaria $\mathrm{x}$ ananassa
Duch.) cv." AlBión ." U.D.C.A Act. \& Div. Cient., 21(1), 61-69. https://doi.org/10.31910/rudca. v21.n1.2018.663

Gilbert, N. (2009). The Disappearing Nutrient. Nature, 461(October), 2000-2002.

Gomez, M. I., Castro, H., Gomez, C. J., \& Gutierrez, O. F. (2010). Optimización de la producción y calidad en cebolla cabezona (allium cepa L.) mediante el balance nutricional con magnesio y micronutrientes ( $b, z n$ y $\mathrm{mn}$ ), valle alto del río chicamocha, boyaca. Agronomia Colombiana, 25(2), 339-348.

Guerrero-Riascos, R. (1995). Fertilización de cultivos en clima medio.

Gutierrez M., L. M., Rodriguez C., L. F., \& Bermudez C., L. T. (2013). Factibilidad de una comercializadora hortícola de economía solidaria en el Distrito de Riego del Alto Chicamocha. Revista Colombiana de Ciencias Hortícolas, 7(1), 62-74.

Hendriksen, K., \& Hansen, S. . (2001). Increasing the dry matter production in bulb onions. Acta Horticulturae, 555, 147-152.

Instituto Colombiano de Normas Técnicas (ICONTEC). NTC1221. frutas y hortalizas frescas. cebolla cabezona (1994).

International Plant Nutrition Institute (IPNI). (1999). Functions of Phosphorus in Plants. Better Crops, 83(1), 6-7.

Larcher, W. (2003). Physiological Plant Ecology. Ecophysiology and Stress Physiology of Functional Groups (4th ed.). Springer-Verlag Berlin Heidelberg.

Maathuis, F. J. (2009). Physiological functions of mineral macronutrients. Current Opinion in Plant Biology, 12(3), 250-258. https://doi. org/10.1016/j.pbi.2009.04.003

Marschner, P. (2012). Mineral Nutrition of Higher Plants (Third edit). Elsevier Ltd.

Ministerio de Agricultura y Desarrollo Rural (MADR). (2018). Estadisticas Agricolas. Área, producción, rendimiento y participación municipal en el departamento por cultivo. Retrieved from http:// www.agronet.gov.co/estadistica/Paginas/default.aspx

Quintana-Blanco, W. A., Pinzón-Sandoval, E. H., \& Torres, D. F. (2017). Efecto de un fosfato termico sobre el crecimiento y producción de fríjol 
(Phaseolus vulgaris L.) cv "ICA Cerinza." Revista U.D.C.A Actualidad \&Divulgación Científica, 20(1), 51-59.

Roberts, T. L., \& Johnston, A. E. (2015). Phosphorus use efficiency and management in agriculture. Resources, Conservation and Recycling, 105, 275-281. https://doi.org/10.1016/j.resconrec.2015.09.013

Singh, S. K., Badgujar, G. B., Reddy, V. R., Fleisher, D. H., \& Timlin, D. J. (2013). Effect of Phosphorus Nutrition on Growth and Physiology of Cotton Under Ambient and Elevated Carbon Dioxide. Journal of Agronomy and Crop Science, 199(6), 436-448. https://doi.org/10.1111/jac.12033
White, P. J., \& Broadley, M. R. (2009). Biofortification of crops with seven mineral elements often lacking in. New Phytologist, 182(1), 49-84. https:// doi.org/10.1111/j.1469-8137.2008.02738.x

Wyngaard, N., Cabrera, M. L., Jarosch, K. A., \& Bünemann, E. K. (2016). Phosphorus in the coarse soil fraction is related to soil organic phosphorus mineralization measured by isotopic dilution. Soil Biology and Biochemistry, 96, 107-118. https:// doi.org/10.1016/j.soilbio.2016.01.022

Zhu, J., Li, M., \& Whelan, M. (2018). Phosphorus activators contribute to legacy phosphorus availability in agricultural soils: A review. Science of the Total Environment, 612, 522-537. https://doi. org/10.1016/j.scitotenv.2017.08.095 
